NOTICE: this is the author's version of a work that was accepted for publication in Composites Science and Technology. Changes resulting from the publishing process, such as peer review, editing, corrections, structural formatting, and other quality control mechanisms may not be reflected in this document. Changes may have been made to this work since it was submitted for publication. A definitive version was subsequently published in Composites Science and Technology, Volume 68, Issue 6, May 2008, DOI: 10.1016/j.compscitech.2007.12.008 


\title{
Deformation mechanisms of MMCs under indentation
}

\author{
A. Pramanik, L. C. Zhang* and J. A. Arsecularatne
}

School of Aerospace, Mechanical and Mechatronic Engineering, University of Sydney, NSW-2006, Australia

\begin{abstract}
This paper investigates the deformation mechanisms of MMCs subjected to microindentation by a spherical indenter using a three-dimensional finite element modeling. It was found that deformation behaviour, hardness and work hardening of MMCs were highly dependant on the location of indentation relative to particles, volume percentage of the particle, and the size ratio of indenter to particle. The hardness of an MMC varied in a complex manner depending on the restriction on the matrix flow by reinforced particles and work hardening of the matrix material. Hardness increased with the increase of volume percentage of reinforced particles and decrease of the size ratio of indenter to particle. Matrix flow due to indentation was highly non-uniform which generated an inhomogeneous strain filed in an MMC. These pose a question that the conventional definition of microhardness is not very appropriate for characterizing MMCs.
\end{abstract}

Keywords: Metal-matrix composites, Plastic deformation, Finite element analysis, Residual stress, Deformation, Hardness testing

\section{Abbreviations}

MMC - Metal matrix composite, FEM - Finite element method, LIRP - Locations of indentation relative to particles, SRIP - Size ratio of indenter to particle, IAP - Indenting exactly above a particle, IMP - Indenting at the middle of four particles

\section{Introduction}

Composite materials have high performance in engineering applications [1]. Metal matrix composites (MMCs), particularly aluminum-based particle/fiber-reinforced composites, have

\footnotetext{
${ }^{*}$ Corresponding author. Tel.: +61 2 93512835; fax: +61 293517060

Email address: zhang@aeromech.usyd.edu.au (L. C. Zhang)
} 
a high strength to weight ratio and wear resistance [2-6], and therefore are increasingly used in automotive and aerospace structures [7]. While the reinforced particles make MMCs different from monolithic materials and induce superior physical properties to MMCs, they also bring about very high tool wear and inferior surface finish when machining MMCs [7]. Thus, in manufacturing, difficulties associated with precision and efficient machining of MMCs have become an important issue [1].

In order to exploit the properties of MMCs, a deeper understanding of their deformation behavior and the interaction between reinforcements and matrix is required. While most of the research to date has been devoted to the development and application of the MMCs, there is little knowledge on the basic mechanisms responsible for their properties [8].

\section{A micro-indentation test is particularly useful for identifying the local effect of particles} under complex stresses similar to machining, one of the most important surfacing techniques for MMCs. A cutting edge in precision machining is sharp and will not interact with many particles. Hence, micro-indentation cannot be replaced by either a traditional tensile or compression test, which generate completely different stress field from machining, or a macro-hardness test, which interact with many particles at the same time and cannot give rise to the detailed micro-interaction between a single particle and its surrounding matrix.

Several studies have used indentation, to investigate the deformation characteristics of MMCs [9-15]. For example, Mussert et al. [16] used nano-indentation experimentally to measure the hardness and elastic moduli profiles of aluminium alloy 6061 reinforced with $\mathrm{Al}_{2} \mathrm{O}_{3}$ in three different heat treatment conditions. Shen et al. [17] explored the macroindentation behavior of MMCs. However, most of the investigations on the properties of MMCs were experimental and did not provide a through analysis of the deformation during loading/unloading for different particle-matrix-indenter arrangements. Because of the complexity of deformation of an MMC and the interaction in the vicinity of contact zone between the indenter and work material, an analytical or experimental method is unable to predict the detailed deformation process [18]. 
The present study will investigate the deformation behaviour of MMCs due to microindentation and its influence on hardness and strain development using the finite element method. In particular, variation of hardness for various loading conditions, size ratio of indenter to particle (SRIP) and particle volume fractions during micro-indentation of MMCs will be investigated for different locations of indentation relative to particles (LIRP). In addition, particle displacement and inhomogeneous deformation behaviour of MMCs will also be investigated.

\section{Modelling}

Two-dimensional FEM analyses on micro-indentation, for instance the one reported in [8], was based on some strong assumptions such as plane-stress deformation, with which the effects of spherical reinforcements cannot be explored. To overcome these limitations and achieve a deeper understanding of the effects of the reinforcement particles, this paper will use a three dimensional finite element model to investigate the influence of LIRP. Two types of indentations will be carried out: (1) indenting exactly above a particle (IAP) (Fig. 1(a)), and (2) indenting at the middle of four particles (IMP) (Fig. 1(b)).

\subsection{Boundary and loading conditions}

The authors' previous analysis [8] has shown that the development of stress/strain fields in a distance from the indentation zone could be considered symmetric around the indenter. Therefore, symmetric boundary conditions were applied on the MMC workpiece to make the three dimensional model size manageable (Fig. 1). Particles were assumed to be uniformly distributed and perfectly bonded with the matrix. The indentation process was considered to be quasi-static. The workpiece was fully fixed on its bottom surface to eliminate rigid body motion. The workpiece dimensions were greater than 3.5 times the indenter radius to avoid boundary effects [18]. The workpiece (Fig.1) was $37.5 \mu \mathrm{m}$ in height, width and length, and the indenter radius was $9 \mu \mathrm{m}$. 
In the loading process, a series of downward increments in the rigid body displacement was imposed on the indenter to induce the indentation into the work material. Particle fracture was not considered in this study.

\subsection{MMC workpiece and indenter}

The MMC work material was a 6061 aluminium alloy reinforced with spherical silicon carbide particles. The reinforcements were treated as an isotropic perfectly elastic material following the generalized Hook's law. The material properties of the particles were: Young's modulus $=400 \mathrm{GPa}$ and Poisson's ratio $=0.17$. The $6061 \mathrm{Al}$ matrix followed a temperatureindependent bilinear kinematic hardening material model and its associated flow rule. The corresponding stress-strain curve given in Fig. 2 was based on the data in [19, 20]. The properties of the matrix were: Young's modulus $=71.6 \mathrm{GPa}$, yield strength $=125 \mathrm{MPa}$, tangential modulus $=1.48 \mathrm{GPa}$ and Poisson's ratio $=0.33$. The diamond indenter was assumed to be linear elastic with modulus of elasticity = $1147 \mathrm{GPa}$ and Poisson's ratio = 0.070. The average coefficient of friction $(\mu)$ obtained by Pramanik et al. [1] during their MMC machining tests, $\mu=0.6$, was used in the present analysis.

It was found from $[8,17,21]$ that the micro-properties of MMCs depend on the LIRP, SRIP, indentation load, volume percentage of particles, and properties of matrix and particles. Hence, in the present investigation these factors were varied to explore their effects. For the investigation of volume percentage of particles, the diameter of the indenter was kept $18 \mu \mathrm{m}$ and particle volume percentages of $10,20 \& 30$ were considered. The diameter of the indenter was varied to obtain different SRIP $(0.8,1.0,1.2 \& 1.4)$ where particle diameter was $18 \mu \mathrm{m}$ and reinforcement volume fraction was $20 \%$. A higher SRIP was obtained by increasing the diameter of the indenter rather than by reducing the size of particles in the MMC because the latter will alter the properties of the MMC.

\section{Results and discussion}

This section will discuss the indentation force-displacement behavior, hardness, strains and their dependence on LIRP, volume percentage of reinforcements and SRIP. 


\subsection{Force-displacement behavior}

The force-displacement curves of micro-indentation of MMCs are presented in Fig. 3. The displacement represents the total displacement of the indenter in the work material, that is, elastic plus plastic. The load displacement curves are related to the elastic modulus and hardness of the work material, but compared with a monolithic material it is more difficult to interpret them in terms of hardness, tensile strength, ultimate strength and modulus of elasticity [22]. For example, the gradient of the force-displacement curves (Fig. 3) varies with indentation load, LIRP, SRIP, etc.

Fig. 3 shows that the load-displacement curves for IAP and IMP bifurcate, and the IAP shows a higher gradient. There are two obvious changes along the load-displacement curves, at points A and B for IAP, and A and F for IMP. This phenomenon was experimentally observed by Mussert et al. [22], attributed simply to the presence of particles. During unloading, curves for IAP and IMP followed similar trend of springback though the gradient of IAP curve is higher than that of IMP curve. At a given load after complete unloading, the residual plastic deformation for IAP is smaller than that of IMP.

Initially the effect of LIRP is negligible for both cases (part OA along the curves). After point A, matrix between indenter and particles experiences high deformation. This results in a trend change of load-displacement curve. For the IMP, the restriction to matrix flow by particle is less and indentation displacement is higher than those for IAP at the same indentation load. Hence, AB shows a higher gradient than AF. At B and F, secondary indentation starts to take place, i.e. reinforcement particles start to act as indenters. Consequently, the force-displacement curves indicate a further increase of gradient.

Higher load bearing capacity of reinforced particles reduces the deformation of the MMC under loading [7, 17]. The total deformation of the MMC for IAP is lower than that of IMP at a given indentation load because in this case particle is located closer to the indenter. For the same reason, in case of IAP, particle carries higher load and matrix deformation is lower than that of IMP. After unloading, the elastic particle will return to its undeformed 
form but plastic matrix will remain deformed. Hence, higher plastic deformation is noted during IMP. These will be further discussed below.

\subsubsection{Effect of volume percentage of particles}

Volume percentage of reinforced particles plays a very important role in the properties of an MMC. Figs. 4(a) \& (b) present the load-displacement responses during loading and unloading for different volume percentages of reinforcements for both IAP and IMP. At the start of the indentations, all the curves show almost the same trend but with further loading, they indicate varying gradient. The gradient increment depends on the volume percentage of reinforcement and LIRP. The higher the volume percentage of reinforcements, the higher the gradient increment. After unloading, lower plastic deformation is noted for the MMC with a higher volume percentage of reinforcement.

A loading curve with higher gradient indicates that a higher load is required for a given depth of indentation, i.e., higher resistance to deformation. Increase of volume percentage of particle means decrease of volume percentage of matrix material and an increase of particle number (for a constant particle size). Hence, an increased number of particles will take part in resisting matrix flow and carrying loads in the composite. Thus it is clear that, with the increase of volume percentage of reinforcements, resistance to deformation increases, i.e., the loading curves show greater gradient. The above mechanism will make an MMC with a higher volume percentage of reinforcements show lower plastic deformation. These are also affected by LIRP due to variation of distance between indenter and particle. It seems that the ceramic particles increase the Young’s modulus and decrease plasticity of MMCs.

\subsubsection{Effect of the SRIP}

Particle size as well as indenter size has significant effect on deformation behavior of MMCs during indentation. The effects of these two parameters can be accounted by considering the size ratio of indenter to particle. Figs. 5(a) \& (b) show the effects of this ratio on the behavior of load-displacement curves for the two types of LIRP. Similar to the effects of volume percentage of reinforcements discussed earlier, at the start of indentation, the 
gradients of load-displacement curves are similar for each case. But with the increase in indentation load, the curves start to deviate at different stages of loading. A loaddisplacement curve corresponding to a higher SRIP shows higher gradient (Figs. 5(a) and (b)). Once again the load-displacement curves for the IAP case show higher gradient compared to those for IMP case. Thus it is clear that resistance of an MMC to deformation increases with the increase of SRIP.

For the ranges of forces/displacements investigated, after unloading, almost constant plastic deformation is noted for all the SRIP considered (Figs. 5(a) \& (b)) but plastic deformation of MMC is higher for IMP than that of IAP. This indicates that, for the tested range of loads, etc., the size of indenter has negligible influence on plastic deformation (depth) of MMCs.

With the increase of indenter diameter, a larger contact area and hence a higher resistance due to reinforced particles is encountered by the indenter at a given indentation load. Therefore, total deformation of an MMC decreases with the increase of SRIP (Fig. 5). Consequently load-displacement curve shows higher gradient at higher SRIP. It is noted that the variation of total deformation of an MMC is low with the variation of SRIP considered in this investigation. Hence, there is not a significant variation of plastic deformation.

\subsection{Hardness}

In the present study, the hardness was determined from the relation [23]

$$
\text { Hardness }=\frac{P}{\left(\frac{\pi D}{2}\right)\left(D-\sqrt{D^{2}-d^{2}}\right)}=\frac{P}{\pi D t}
$$

where $P=$ applied load, $D=$ diameter of indenter, $d=$ diameter of the indentation mark after complete unloading and $t=$ depth of the indentation mark after complete unloading.

\subsubsection{Effects of indentation load on hardness}

Hardness of a material obtained by indentation is a measure of its resistance to plastic deformation. Micro-hardness of an MMC, compared to a monolithic material may show 
greater dependency on indentation load because of its inhomogeneous deformation behavior due to the presence of reinforcement particles. For the two types of LIRP, variation of hardness at various stages of loading is obvious.

The indentation loads selected correspond to points A, B, C, F and G on the loaddisplacement curves in Fig. 3. These points were selected to investigate the effect of gradient changes of load-displacement curve on the hardness of an MMC. Some high loads beyond points $\mathrm{C}$ and $\mathrm{G}$ were also considered to observe hardness variation over a wider range of loads. Indentations were performed with loads at corresponding points and then it was unloaded completely to obtain corresponding $d$ and $t$ values. The hardness values corresponding to these loads were then calculated using Eq. (1). These results are shown in Fig. 6 where hardness of the MMC is found to increase at different stages of loading at different rates. The variation of hardness can be explained as follows.

For indentation with a very low load, the effect of LIRP is small (point A in Fig. 6). With the increase of indentation load, the hardness of the MMC continues to increase due to higher resistance to plastic deformation of matrix material. For IAP, the resistance to plastic deformation is much higher than that for IMP case due to the greater resistance by the particles on the matrix flow in the former (described in Sec. 3.1). Hence the rate of increase of hardness with increase of indentation load is higher for the IAP case, i.e., gradient of AB is higher than that of AF. At points B and F, secondary indentation by particles near the indenter takes place, which causes a further increase of hardness. The increase of hardness is much higher for IAP case (BC) than that of IMP (FG). Then the matrix below the particle (secondary indenter) starts to deform significantly and secondary indentation occurs with the increase of loading. The secondary indentation and associated additional restriction on matrix flow further increases the hardness (CM and GP in Fig. 6) depending on the matrix material properties, particle concentration, size and shape [21]. After points $\mathrm{M}$ and $\mathrm{P}$, constraint to matrix flow applied by the particles around the secondary indenter becomes significant. At this stage primary and secondary particles (those below the primary particle) 
come closer. This further restricts the matrix flow, resulting in an increase in the local hardness [24]. Therefore, hardness continues to increase with loading. Since the reinforcement particles are much stiffer than the matrix, they carried a significant fraction of load during indentation.

It is interesting to note that there are four stages of hardness increase during an indentation of an MMC. These are (i) initial resistance to indentation (AB, AF) (ii) start of secondary indentation (BC, FG, until lower surface of particle completely takes part in indentation) (iii) secondary indentation (CM, GP, where the strain field is not extended to particles) and (iv) tertiary deformation or stabilizing stage (MN, PQ, where the matrix flow is restricted by particles). Clearly the initial resistance to deformation is higher for IAP than that of IMP (slope of $\mathrm{AB}>$ slope of $\mathrm{AF}$ ) since a particle is located near the indenter in the former. During the secondary indentation, the rate of increase of hardness is slightly higher for IMP, because for this case the matrix between indenter and particles offers a greater resistance to deformation (because of shorter distance between them and higher MMC volume involved in deformation) compared to the matrix between indenter to particles for IAP. At the stabilizing stage, hardness for both cases increases at a similar rate with the increase of indentation load.

Leggoe et al. [15] experimentally showed that the presence of reinforcement particles restricts matrix flow in an MMC resulting in a higher hardness during indentation. This phenomenon was also noted in the present investigation as described above. The higher the indentation force/displacement, the higher is the particle concentration underneath the indentation $[24,25]$. The increase of hardness with the increase of load in the stabilizing stage for a particle reinforced MMC can be attributed to the localized increase in particle concentration directly underneath the indenter during a hardness test [17].

\subsubsection{Effects of volume percentage \& SRIP}

To investigate the effect of reinforcement volume percentage and SRIP on hardness, a constant indentation load, $0.308 \mathrm{~N}$, was used on the basis of sufficient deformation. Fig. 7 
presents the effect of reinforcement volume percentage. It is clear that hardness of an MMC increases with the increase of volume percentage of reinforcement for both cases. But the rate of increase is much higher for IAP. As discussed earlier, the hardness for IAP is higher. It seems that further addition of reinforcement particles top ups the hardness over that of IMP.

It was found from load-displacement curves described in Sec. 3.1.1 that an MMC with a higher percentage of reinforcements has higher resistance to deformation and lower plastic deformation (Figs. 4(a) \& (b)). Hence, MMCs with higher percentages of reinforcements show higher hardness.

Fig. 8 shows the influence of SRIP. With the increase of SRIP, hardness is found to decrease. An interesting feature is that the rate of decrease with SRIP is similar for the two LIRP cases.

As discussed in Sec. 3.1.2, during indentation, with the increase of SRIP, MMCs show little increase of total deformation during loading but no significant variation in plastic deformation (depth) after unloading. Since, with the increase of SRIP, plastic deformation of an MMC does not vary but the diameter of indenter increases and the hardness decreases.

\subsection{Development of strain fields}

The contours of von Mises total strain at different points on load-hardness curves (Fig. 6) are presented in Figs. 9 and 10. These explain the deformation mechanism of an MMC and hardness changes over the range of loading considered. Strains developed in the indenter and reinforced particles during indentation process are negligible compared to those of matrix material because of high modulus of elasticity of the indenter and particles. Hence, only the strain development in the matrix material is considered here. This will also include details at the particle-matrix and indenter-matrix interfaces.

\subsubsection{Indentation above a particle}

Fig. 9 depicts the variation of the von Mises total strain in the MMC for IAP. At the start of loading, the strain distribution is not uniform but in a layered pattern with the maximum 
strain zone near the contact interface (Fig. 9 (a)). There is no significant strain at the particlematrix interface and hence the effect of LIRP is small. This stage can be compared with the situation at point A in Figs. 3 \& 6. With further loading, the volume of strain field increases and significant strains are noted at particle-matrix interface (Fig. 9 (b)). At this stage the maximum stain zone moves down towards the particle and, the matrix between indenter and particle is highly strained. This situation can be compared with that of point B in Figs. 3 \& 6 . As the loading continues, significant deformation of matrix under the particle starts, which causes secondary indentation in the matrix by the reinforced particle (Fig. 9 (c)). The resulting secondary deformation continues until the effect of secondary particle becomes significant. Fig. 9(d) represents the strain state at point $M$ in Fig. 6. The strain field during tertiary deformation at a point between $\mathrm{M}$ and $\mathrm{N}$ is presented in Fig. 9 (e). In this range the particle constrains the matrix flow.

\subsubsection{Indentation between particles}

The contours of strain for IMP are presented in Fig. 10. At the beginning of loading, the strain field is spherical and in a layered pattern (Fig. 10(a)). The effect of LIRP is negligible at this stage which can be compared to strain state at point A in Figs. 3 \& 6. Up to this stage, the mechanism of strain field generation for this case is almost similar to that described in Section 3.3.1; but the developed strains at the corresponding points are considerably lower than those of IAP. As the loading progresses, the volume covered by the strain field increases and reaches the boundaries of particles (Fig. 10(b)). It is interesting to note that the point with the maximum straining initially appears near the indenter (Fig. 10(a)), but with continued loading it moves towards the particle and reaches the particle-matrix interface. Secondary indentation then starts and the strain state corresponds to that at point F (Fig. 10(b)). The same phenomenon was noted for the IAP. With further loading, matrix material passes through secondary and tertiary deformation states as shown in Figs 10(c) \& (d) respectively in a manner similar to that described in the Section 3.3.1. These results 
demonstrate the effect of reinforcement particles on deformation of matrix material and hardness of an MMC.

From the above discussion, it can be concluded that initial yielding of matrix occurs near the indentation interface and then extends to the particle-matrix interface(s) through the matrix. The particles are well discerned in the strain fields because no plasticity exists in highly elastic particles. The presence of discrete particles dramatically fragments the plastic field and causes extreme inhomogeneous deformation and flow of matrix in the MMC. Thus localized deformation of the MMC can be expected after indentation. The amount of deformation of matrix material depends on the LIRP. These are in agreement with the experimental observations [26].

Similar to a monolithic material subjected to indentation [27], yielding in an MMC also occurs first at a small distance beneath the indentation interface (Figs. 9 (b) \& 10 (b)).

\subsection{Work hardening during indentation of MMC}

It is noted that considerable stresses develop in the matrix due to significant, nonuniform deformation in the vicinity of the indentation. At the initial loading, (e.g. at point A in Fig. 3) the highest von Mises stresses developed for IAP and IMP are 153 and $141 \mathrm{MPa}$ respectively. The yield stress is $125 \mathrm{MPa}$ for aluminum alloy matrix considered in this investigation. Thus considerable work hardening of matrix material occurs from initial loading. The stresses and strains in the matrix continue to increase with further loading which will cause further hardening of the matrix material. The level of strain and its rate of increase depends on LIRP, indentation load etc. and thus will influence work hardening. Higher strains and higher work hardening occurs in the matrix between indenter and particles (e.g. Figs. 9 (b-c) and Figs. 10 (b-c)) or between particles near indentation (Fig. 9

(e)), due to the restriction of matrix flow by particles. The deformation behavior of aluminium alloy (Curve 1) and MMC with different volume percentages of reinforcements at various loads were given in Fig.4. These curves clearly show the high work hardening rate of MMC (much higher gradient of load/displacement curves) compared to aluminium alloy. 
This is in agreement with the experimental observations by Li et al [32]. These non-uniform strains and work hardening cause the variation of hardness observed for IAP and IMP in Fig. 6.

\subsection{Reinforcement displacement and interaction}

Figs. $9 \& 10$ reveal that, with the advancement of indenter, particle(s) underneath are displaced with surrounding matrix and the matrix in between the indenter and particle is highly squeezed. It is noted that the inter-particle spacing is reduced in the region below the indentation interface and the distance between indenter and particle is decreased which causes interaction between them with further loading. As the particles are hard, frequent interactions will cause abrasive wear to the indenter. This can be a main reason for high tool wear during machining of MMCs [28-31].

\section{Conclusions}

Due to the presence of reinforcements, MMCs behave very differently compared to monolithic metals during deformation. While micro-indentation is simple to carryout, the $\underline{\text { test results at low indentation loads should be interpreted carefully because the microscopic }}$ deformation processes are complex. The present investigation has shown that:

(i) The ceramic particles increase the MMC's ability to resist deformation, but this is highly dependant on the location of indentation relative to particles, volume percentage of particles, size ratio of indenter to particle and applied load. Consequently, these parameters affect hardness of MMCs.

(ii) The mechanisms responsible for the anisotropy of MMCs are: varied restriction to matrix flow by particles and non-uniform work hardening of MM depending on the combination of above mentioned parameters.

(iii) The micro-indentation test under low load cannot give to a consistent measure of the hardness of MMCs.

\section{Acknowledgements}


The authors wish to thank the Australian Research Council for financial assistance. AP is under IPRS and IPA scholarships.

\section{References}

[1] A. Pramanik, L. C. Zhang, J. A. Arsecularatne, "Prediction of cutting forces in machining of metal matrix composites”, International Journal of Machine Tools and Manufacture 46(2006) 1795-1803.

[2] Z. F. Zhang, L. C. Zhang, and Y.W. Mai, "Particle effects on friction and wear of aluminium matrix composites”, Journal of Materials Science, 30(23) (1995) 5999-6004.

[3] C. Yan and L. C. Zhang, "Single-Point Scratching of 6061 Al Alloy reinforced by Different Ceramic Particles”, Applied Composite Materials, 1(1995) 431-447.

[4] Z. F. Zhang, L. C. Zhang, and Y.W. Mai, "Wear of ceramic particle-reinforced metalmatrix composites, part I wear mechanisms”, Journal of Materials Science, 30(8) (1995) 1961-1966.

[5] Z. F. Zhang, L. C. Zhang and Y.W. Mai, "Modeling steady wear of steel/ $\mathrm{Al}_{2} \mathrm{O}_{3}-\mathrm{Al}$ particle reinforced composite system”, Wear, 211(2) (1997) 147-150.

[6] Z. F. Zhang, L. C. Zhang, and Y.W. Mai, "Wear of ceramic particle-reinforced metalmatrix composites, part II a model of adhesive wear”, Journal of Materials Science, 30(8) (1995) 1967-1971.

[7] A. Pramanik, L. C. Zhang, J. A. Arsecularatne, “An FEM investigation into the behaviour of metal matrix composites: tool-particle interaction during orthogonal cutting”, International Journal of Machine Tools and Manufacture, 47 (2007) 1497-1506.

[8] A. Pramanik, L. C. Zhang, J. A. Arsecularatne, "Micro-indentation of metal matrix composites-an FEM analysis”, Key Engineering Materials, 340-341 (2007) 563-570.

[9] T. G. Nieh and R. F. Karlak, "Aging characteristics of $B_{4}$ C-reinforced 6061-aluminum” Scripta Metallurgica et Materialia, 18 (1984) 25-28.

[10] S. Suresh, T. Christman and Y. Sugimura, “Accelerated aging in cast al alloy-SiC particulate composites”, Scripta Metallurgica et Materialia, 23 (1989) 1599-1602. 
[11] K. K. Chawla, A. H. Esmaeili, A. K. Datye, and A. K. Vasudevan, “Effect of homogeneous/heterogeneous precipitation on aging behavior of SiCp/Al 2014 composite”, Scripta Metallurgica et Materialia, 25 (1991) 1315-1319.

[12] T. Christman and S. Suresh, "Microstructural development in an aluminum alloy-SiC whisker composite”, Acta metall. 36(7) (1988) 1691-1704.

[13] P. Appendino, C. Badini, F. Marino and A. Tomasi, “6061 Aluminum alloy-SiC particulate composite: a comparison between aging behaviour in T4 and T6 treatments”, Materials Science arid Engineering, A 135 (1991) 275-279.

[14] G. Cseh, J. Bar, H. J. Gudladt, J. Lendvai and A. Juhasz, "Indentation creep in short fiber-reinforced metal matrix composite”, Material Science and Engineering, A272 (1999) 145-151.

[15] J. W. Leggoe, X. Z. Hu, M. V. Swain, M. B. Bush, “An ultra-micro indentation investigation of aspects of the fracture process in particulate reinforced metal matrix composites”, Scripta Metallurgica et Materialia, 31(5) (1994) 577-5825.

[16] K. M. Mussert, W. P. Vellinga, A. Bakker, S. Van Der Zwaag, “A nano-indentation study on the mechanical behaviour of the matrix material in an $\mathrm{AA6061-} \mathrm{Al}_{2} \mathrm{O}_{3} \mathrm{MMC}$ ”, Journal of materials science, 37 (2002) 789-794.

[17] Y. L. Shen, J. J. Williams, G. Piotrowski, N. Chawla and Y. L. Guo, “ Correlation between tensile and indentation behavior of particle-reinforced metal matrix composites: An experimental and numerical study”, Acta mater. 49 (2001) 321-3229.

[18] L. Zhang, M. Mahdi, “The plastic behaviour of silicon subjected to micro-indentation”, Journal of materials science, 31 (1996) 5671-5676.

[19] G. Meijer, F. Ellyin and Z. Xia, “Aspects of residual thermal stress/strain in particle reinforced metal matrix composites”, Composites: Part B 31 (2000) 29-37.

[20] S. G. Long, Y. C. Zhou, “Thermal fatigue of particle reinforced metal-matrix composite induced by laser heating and mechanical load”, Composites Science and Technology, 65 (2005) 1391-1400. 
[21] J. W. Leggoe "Determination of the elastic modulus of microscale ceramic particles via nanoindentation”, Journal of Materials Research, 19 (8) (2004) 2437-2447.

[22] K. M. Mussert, W. P. Vellinga, A. Bakker, S. Van Der Zwaag, “A nano-indentation study on the mechanical behaviour of the matrix material in an $\mathrm{AA6061-}-\mathrm{Al}_{2} \mathrm{O}_{3} \mathrm{MMC}$, Journal of materials science, 37 (2002) 789-794.

[23] G. E. Dieter, “Mechanical Metallurgy”, SI Metric Edition, McGraw-Hill Book Company (UK) Limited, 1988.

[24] R. Pereyra, Y. -L. Shen, “Characterization of particle concentration in indentationdeformed metal-ceramic composites”, Material Characterization 53 (2004) 373-380.

[25] Y. -L. Shen, N. Chawla, “On the correlation between hardness and tensile strength in particle reinforced metal matrix composites”, Materials Science and Engineering A: Structural Materials: Properties, Microstructure and Processing, 297 ( 1-2) (2001) 44-47

[26] K. Unterweger, O. Kolednik, “The local deformation behaviour of MMCs -an experimental study”, Material Research and Advanced Techniques, 96 (9) (2005) 10631068.

[27] K. L. Johnson, “Contact Mechanics”, Cambridge university press, 1985.

[28] P. J. Heath, “Developments in applications of PCD tooling”, Journal of Material Processing Technology, 116 (2001) 31-38.

[29] N. P. Hung, N. L. Loh, V. C. Venketesh, “Machining of metal matrix composites in Machining of ceramics and composites”, in: Machining of ceramics and composites, S. Jahanmir, M. Ramulu, P. Koshy (Eds.), (1999), pp. 295-356.

[30] N. P. Hung, C. H. Zhong, “Cumulative tool wear in machining metal matrix composites Part 1: modeling”, Journal of Material Processing Technology, 58 (1996) 109-113.

[31] N. Tomac, K. Tonnessen, “Machinability of particulate aluminium matrix composites”, Ann. CIRP (1992) 55-58. 


\section{Captions for figures}

Fig. 1. 3D model of MMC for micro-indentation

Fig. 2. The stress versus strain curve for 6061 aluminium matrix

Fig. 3. Load displacement curves for different LIRP (SRIP = 1 and particle volume $\%=20$ )

Fig. 4. Effects of reinforced particle volume percentage on load-displacement curves (SRIP = 1)

Fig. 5. Effects of SRIP on load-displacement curves (particle volume \% = 20)

Fig. 6. Effects of indentation load on hardness of MMC (SRIP = 1 and particle volume $\%=20)$

Fig. 7. Effect of reinforcement volume percentage on hardness of MMC (SRIP $=1$, particle volume $\%=20$ and indentation load $=0.308 \mathrm{~N}$ )

Fig. 8. Effect of SRIP on hardness of MMC (particle volume $\%=20$ and indentation load $=0.308$

N)

Fig. 9. Von Mises total strain in the matrix for IAP (SRIP $=1$ and particle volume $\%=20)$

Fig. 10. Von Mises total strain in the matrix for IMP (SRIP $=1$ and particle volume $\%=20$ )

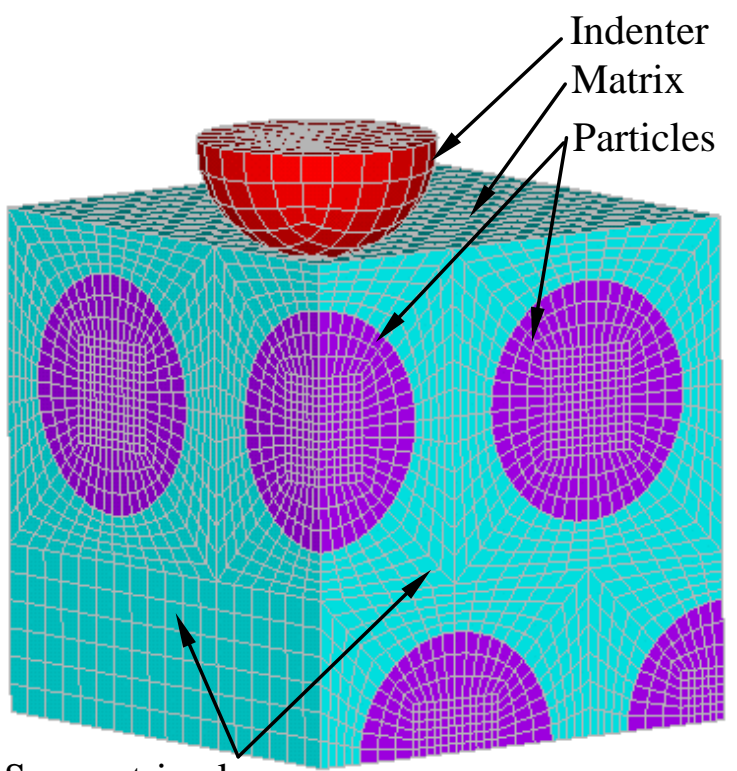

Symmetric planes

(a) IAP

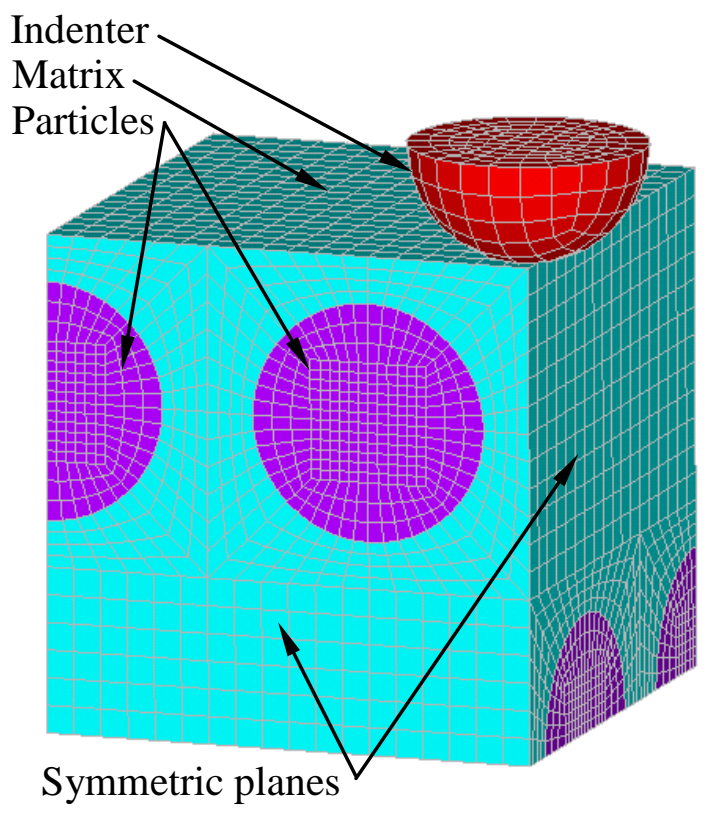

(b) IMP

Fig. 1 


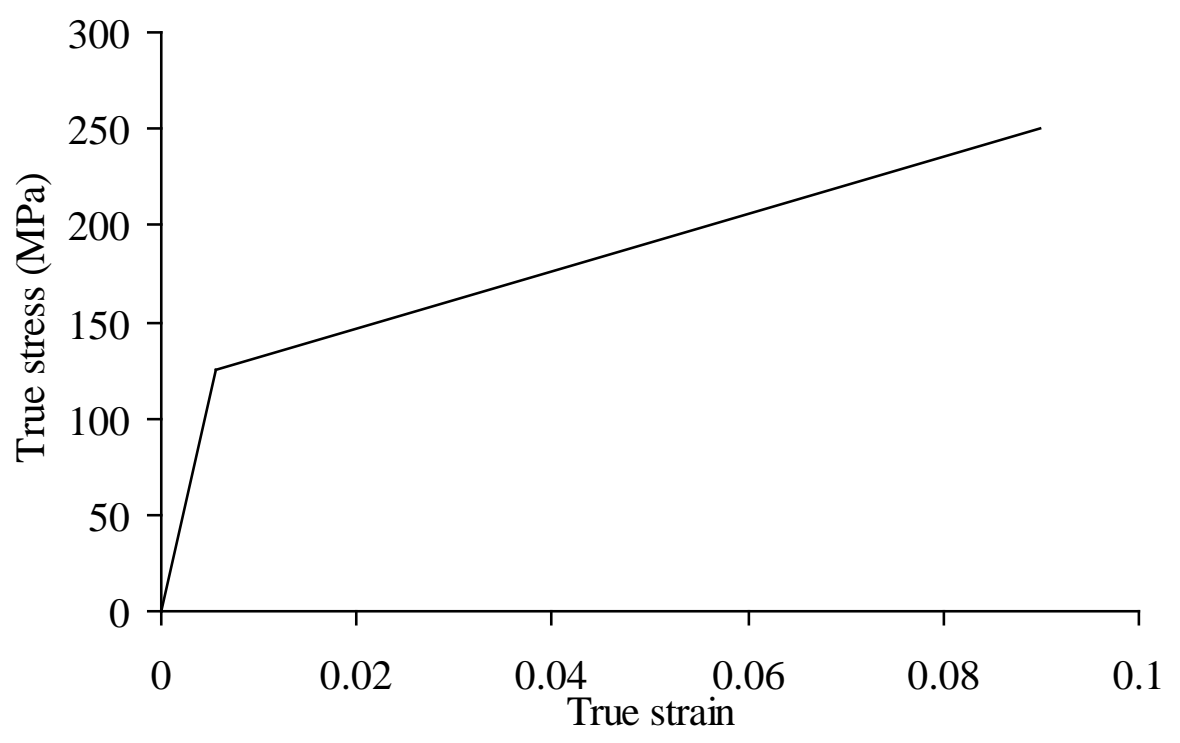

Fig. 2

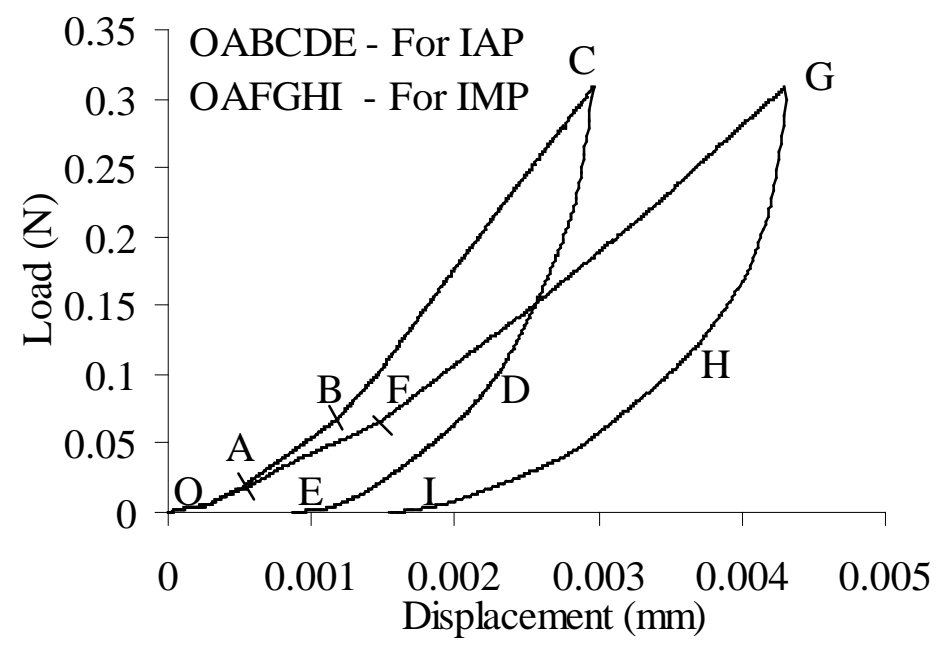

Fig. 3 


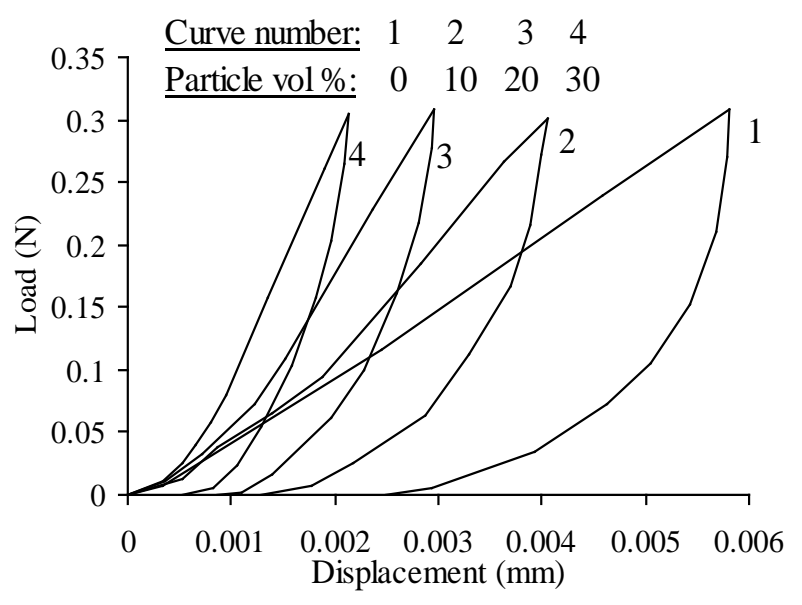

(a) IAP

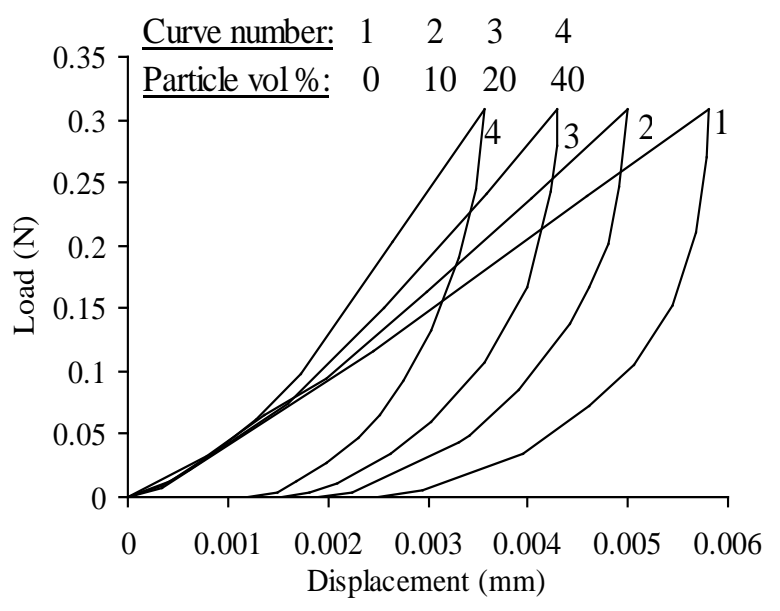

(b) IMP

Fig. 4

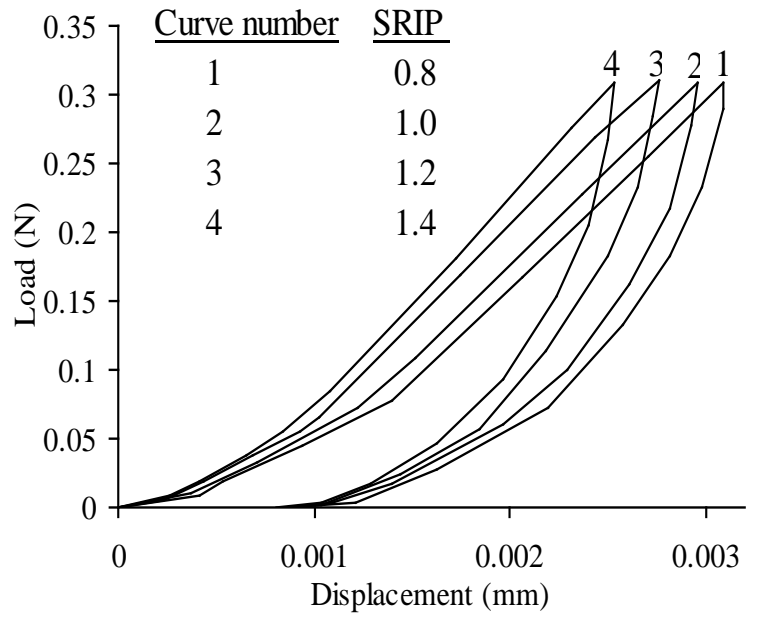

(a) IAP

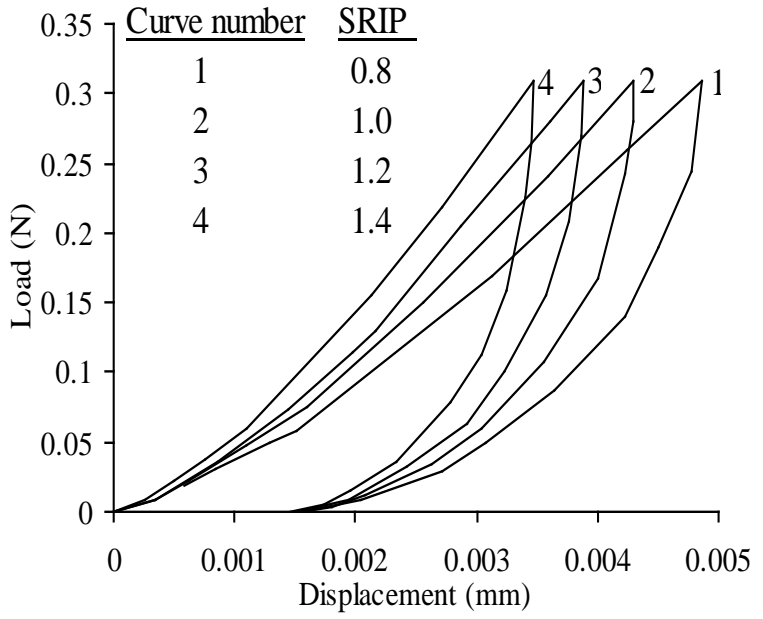

(b) IMP

Fig. 5 


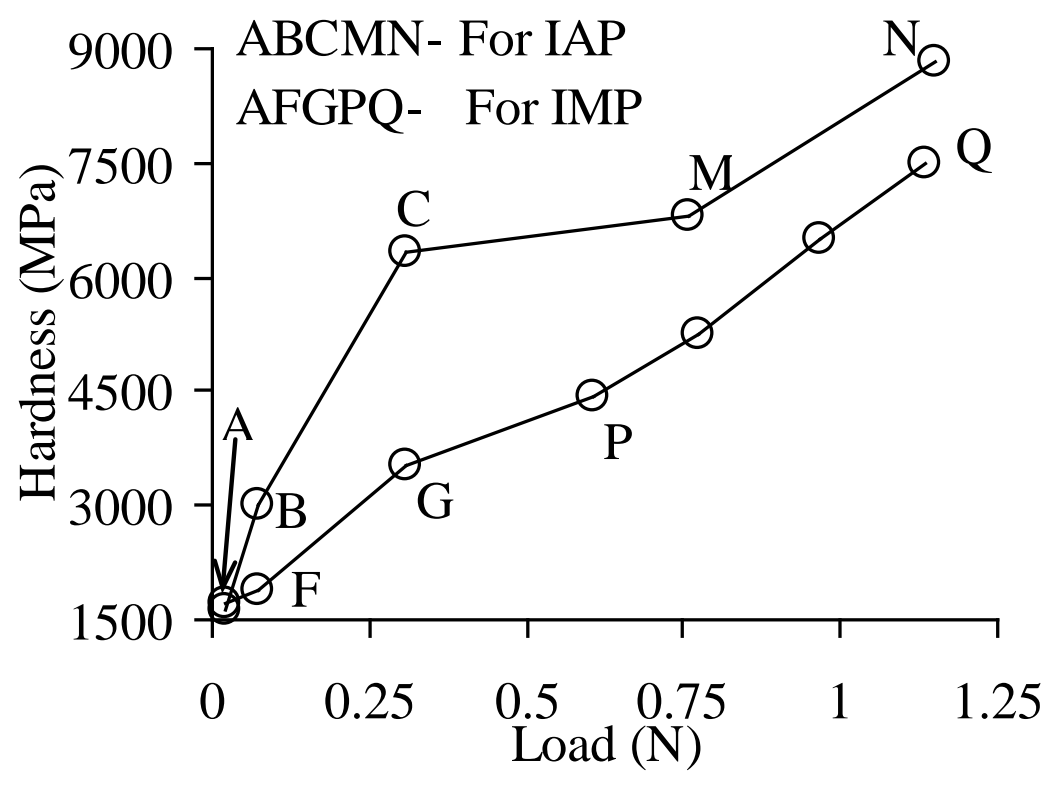

Fig. 6

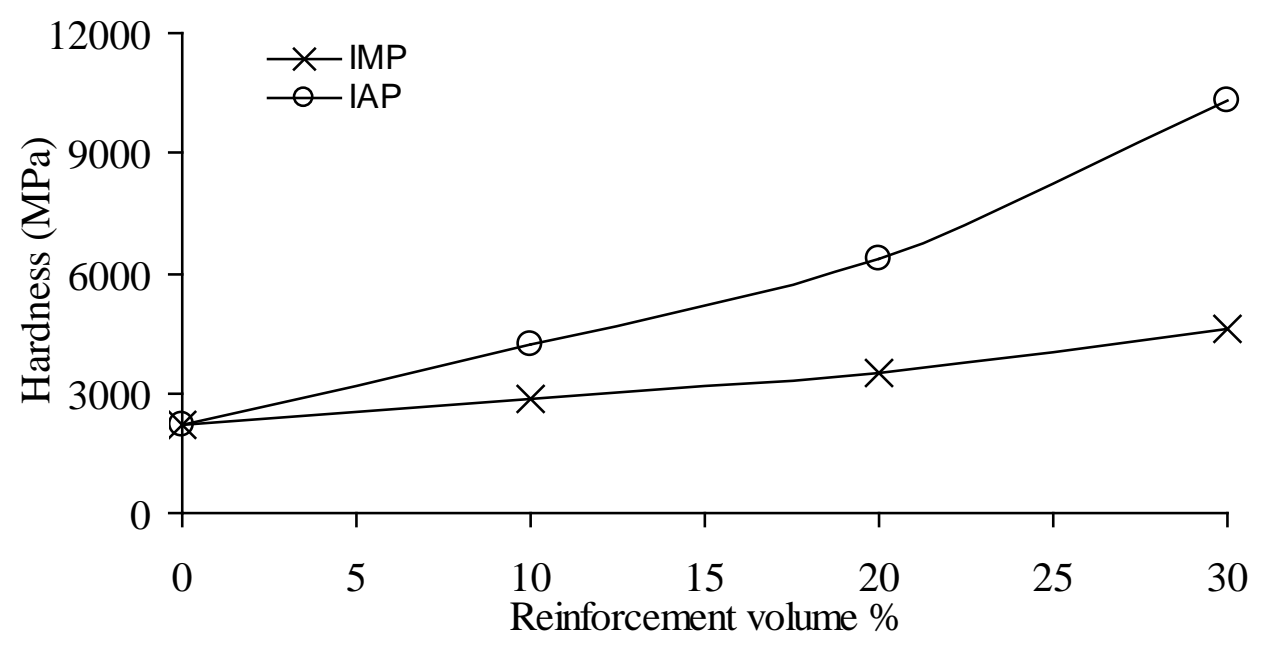

Fig. 7 


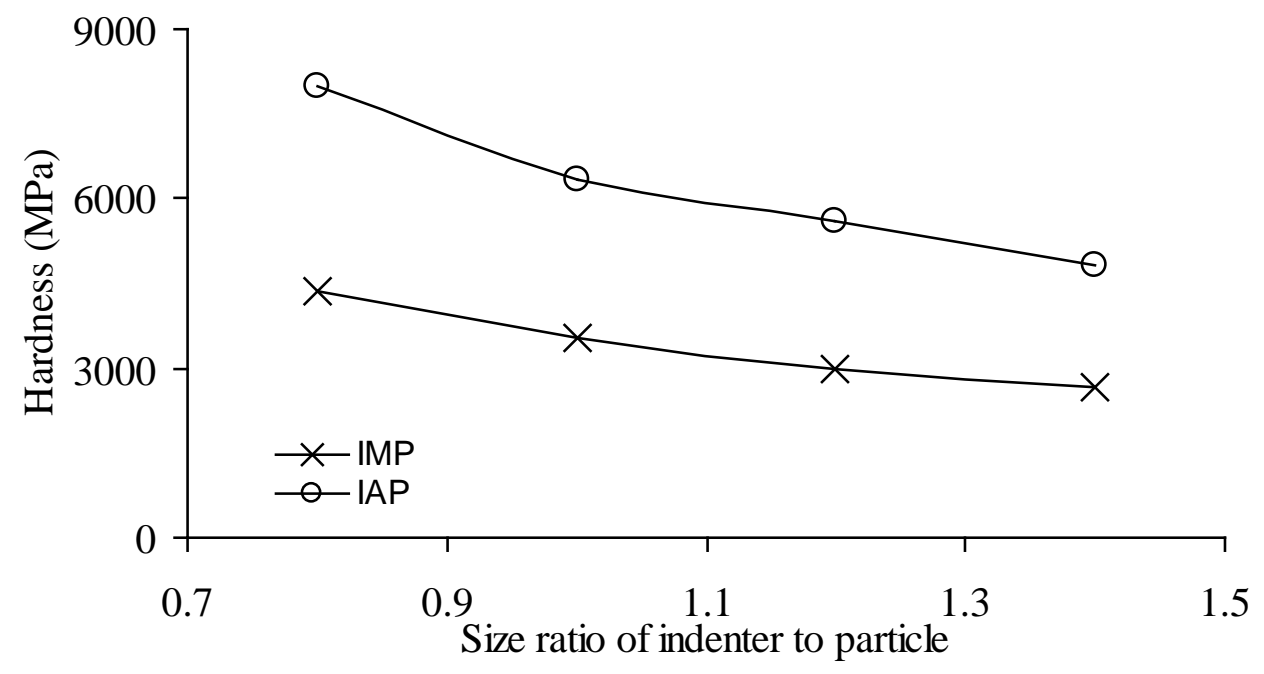

Fig. 8

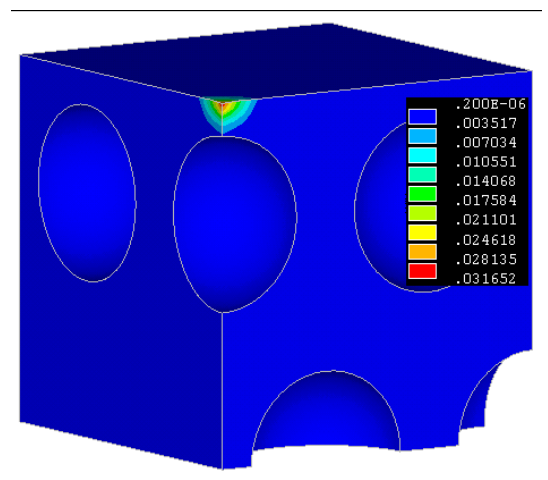

(a) At point A in Fig. 6

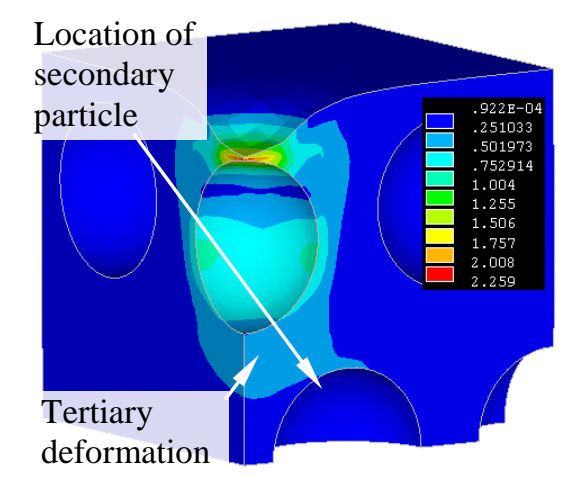

(d) At point $\mathrm{M}$

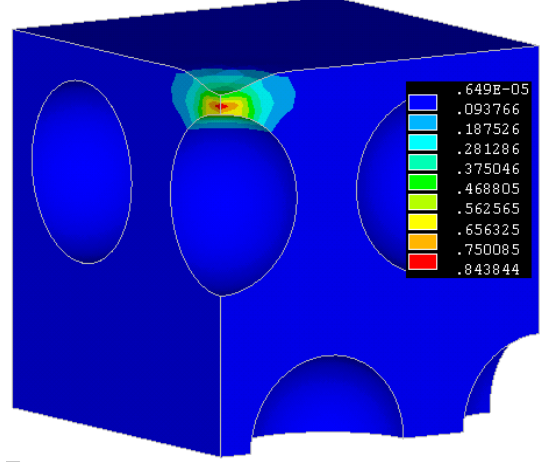

(b) At point $\mathrm{B}$

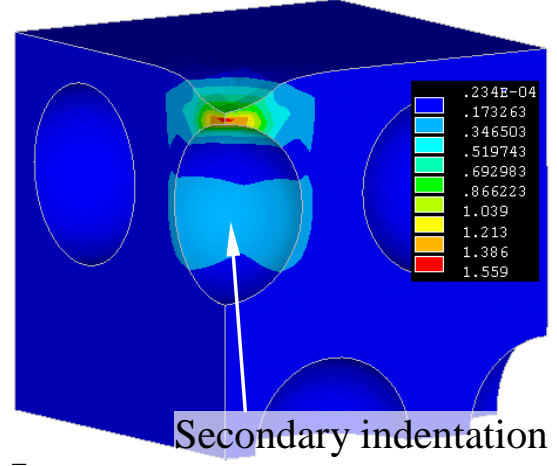

(c) At point C

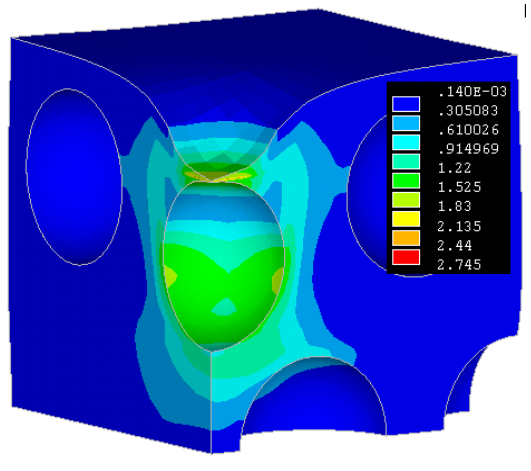

(e) In between points $M \& N$

Fig. 9 


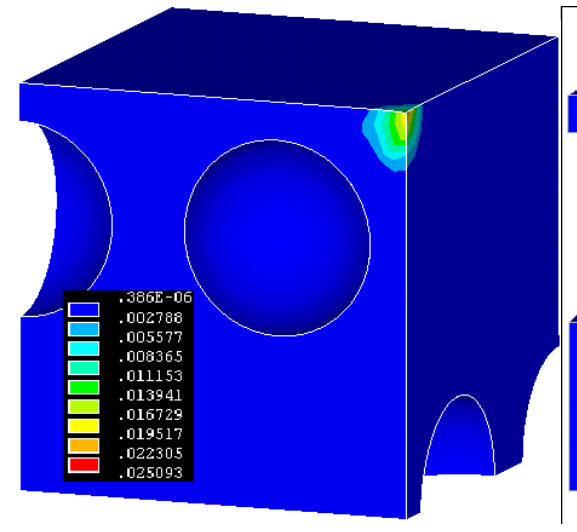

(a) At point A in Fig. 6

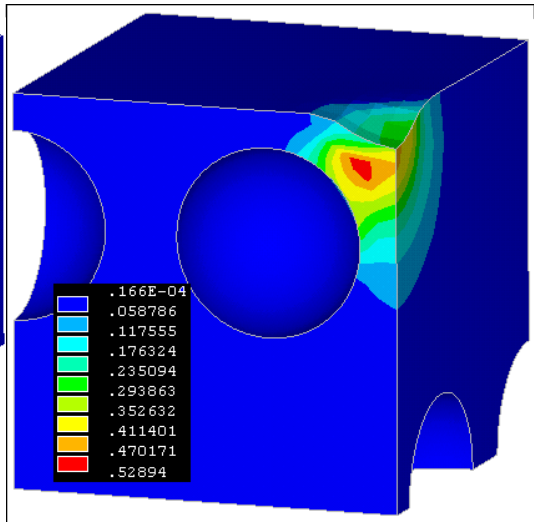

(b) At point $\mathrm{F}$

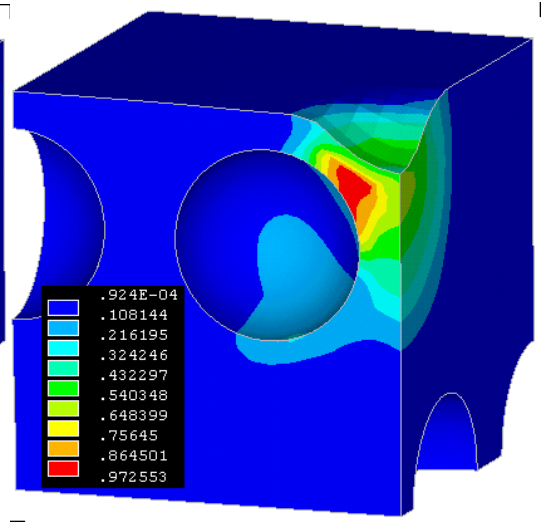

(c) At point G

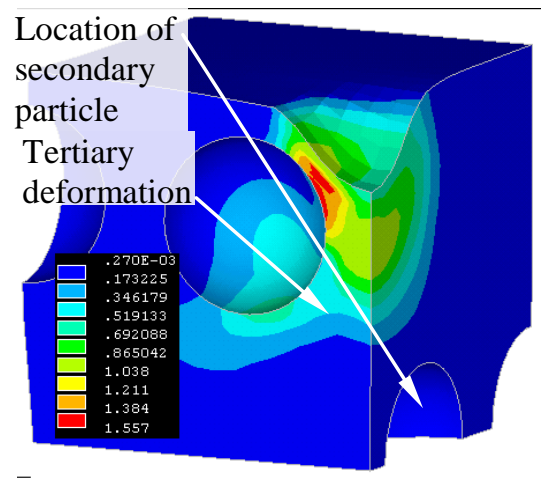

(d) At point $\mathrm{P}$

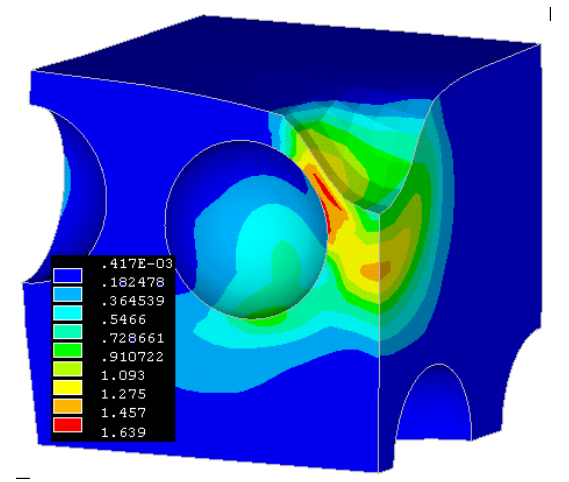

(e) In between $P \& Q$

Fig. 10 